\title{
Palliative care for patients in the USA with amyotrophic lateral sclerosis: current challenges
}

This article was published in the following Dove Press journal:

Nursing: Research and Reviews

24 November 2015

Number of times this article has been viewed

\section{Gail Houseman' \\ Mary Kelley ${ }^{2}$}

'The ALS Association Greater Philadelphia Chapter, Ambler, PA, USA; ${ }^{2}$ Department of Neurology, ALS Center at Penn Medicine, Philadelphia, PA, USA
Correspondence: Gail Houseman The ALS Association Greater Philadelphia Chapter, 32I Norristown Road, Suite 260, Ambler, PA 19002, USA

$\mathrm{Tel}+\mathrm{I} 2156435434$ ext 23

Fax + I 2156439307

Email gail@alsphiladelphia.org
Abstract: Amyotrophic lateral sclerosis (ALS) is a motor neuron disease that results in eventual paralysis of all voluntary muscles. Cognitive impairment may be a co-occurring condition with the ALS patient. Palliative care, which involves symptom management, is the most utilized treatment of choice. Managing the symptoms of ALS can be challenging. This paper provides experience-based facts on daily care provision in the USA and some practical guidelines.

Keywords: amyotrophic lateral sclerosis, ALS, palliative care, challenges, symptom management

\section{What is ALS?}

Amyotrophic lateral sclerosis (ALS) is a progressive, neuromuscular disorder that involves the degeneration of both the upper and lower motor neurons, resulting in the eventual and complete paralysis of all voluntary muscles. This includes not only the muscles of the arms, legs, neck, and trunk, but also the muscles used to speak, chew, swallow, and breathe. There is no known cause of ALS. Riluzole is the only US Food and Drug Administration (FDA)-approved medication that has been demonstrated to slow the progression of the disease "to a modest degree", according to the recent American Academy of Neurology (AAN) Practice Parameter update (2009). ${ }^{1}$ While the speed of progression varies, the average life expectancy after diagnosis is approximately $2-5$ years ${ }^{2}$ the usual cause of death is respiratory failure.

\section{Occurrence - who gets ALS?}

ALS occurs throughout the world in all cultures. The incidence of ALS in the USA is two per 100,000 people. ${ }^{3}$ Recent data from the United States of America's National Registry of USA suggest that the prevalence of ALS in the US population is 3.9 cases of ALS per 100,000 persons and that the disease is more common among white males, non-Hispanics, and persons aged 60-69. ${ }^{4}$ Age of disease onset can occur from the teens to the 90s, however. US military veterans are twice as likely to develop ALS compared to the general population. ${ }^{5}$ Approximately $5 \%-10 \%$ of all cases of ALS are familial; offspring from a parent with the familial form of ALS have a $50 \%$ chance of inheriting the disease.

\section{Cognitive component}

Researchers in the last decade and a half have established that approximately $50 \%$ of all people with ALS have some form of cognitive impairment (CI) ${ }^{6,7}$ This impairment 
ranges from mild CI to severe (frontotemporal degeneration or FTD). The CI may precede the onset of ALS symptoms or coincide with the ALS symptoms. Memory and visuospatial function impairment may occur, but are regarded as less common cognitive changes in ALS. ${ }^{8}$ Rather, in most studies, the most affected cognitive domains are executive function, language, and verbal fluency. ${ }^{6,7}$ People with both ALS and CI have been shown to have shorter survival times, ${ }^{9}$ for reasons that are not clear. While the behavioral variant is the most common variant associated with the ALS/FTD population in approximately $5 \%-10 \%$ of all cases, CI may include any or all of the variants, as shown in Table $1 .{ }^{10-12}$

\section{Palliative treatment of ALS}

Ideally, people with ALS will be active participants in planning the type of care that they, and their loved ones, choose. Aggressive-type treatment would mean that the person with ALS would undergo a tracheostomy and (eventually) require invasive mechanical ventilation. Most people with ALS (90\%-95\%) in the USA, however, do not choose to be fully vented via a tracheostomy. ${ }^{13,14}$

Palliative care, which may include hospice care, is the most (patient) chosen and utilized treatment of choice for people in the USA with ALS. Palliative care in the management of the ALS patient involves addressing and treating the (many) problematic symptoms inherent with the illness, with the goal of providing both comfort and maximum quality of life (QoL).

Table I Variants of cognitive impairment association with ALS

Behavioral dysfunction - potential problems with:

- Loss of sympathy or empathy: diminished response to other people's needs and feelings.

- Apathy or inertia: loss of interest, drive, or motivation.

- Negative interactions with people, loss of awareness of social rules, angry outbursts, impulsivity, loss of emotional connection.

- Increased undesired behaviors, decrease in activity, apathy.

- Day-to-day problematic behaviors, such as excessive spending, perseverating, compulsive, repetitive, or ritualistic behaviors.

- Change in eating behavior: food fads, not eating, stuffing food.

- Neglecting personal hygiene.

Executive dysfunction - potential problems with:

- Planning, sequencing, prioritizing, multitasking, self-monitoring, and correcting behavior.

- Reasoning and insight.

- Verbal fluency.

Language dysfunction (not related to dysarthria) - problems with:

- Word finding, grammatical errors, using phrases in place of sentences.

- Empty speech, impaired understanding of word meaning and/or ability to recognize objects or faces.

- Verbal fluency.

Abbreviation: ALS, amyotrophic lateral sclerosis.
Care may be provided in an office or an outpatient setting, in the patient's home or via telesupport by a variety of health care professionals, including the nurse. At an ALS Association (ALSA)-certified center in the USA, people with ALS and their loved ones typically are seen between 1 and 6 months by a variety of health care professionals (listed in Table 2).

In the USA, people with ALS who elect for palliative versus aggressive treatment may choose to enroll in hospice care. Hospice is generally recommended when the primary care provider believes the person with ALS has 6 months or less to live, and is interested in this type of care. Hospice care is similar to interdisciplinary care; hospice utilizes a team approach to provide comfort to patients by attempting to manage problematic symptoms.

Unfortunately, symptom management in the ALS population has not been well-studied. The 2009 AAN (ALS) Practice Parameter update group found that, in general, there is a paucity of evidence-based research specific to treating symptoms of ALS. ${ }^{15}$ In addition, the authors noted that available and efficacious treatments are, again in general, underutilized by the ALS population. ${ }^{1}$

The remainder of this paper will address specific problematic, and at times, challenging symptoms experienced by the ALS population, along with possible treatments for each symptom. Suggested interventions are based on those AAN suggestions that have been found to be efficacious, along with the combined experience of the author's participation in caring for people with ALS at a multidisciplinary, nationally certified ALS clinic in Philadelphia, PA, USA. Nursing considerations and caregiver issues, among this population, will also be addressed.

Table 2 Interdisciplinary team members associated with an ALSA-certified ALS center in the USA

- Neuromuscular specialist - neurologist who specializes in neuromuscular disorder

- Nurse manager/coordinator of the clinic and care

- Physical and occupational therapists

- Speech and language pathologists

- Dietitian

- Social worker

May also include the following clinicians:

- Respiratory therapist

- Mental health professional (nurse, therapist)

- Pulmonologist

- Communication specialist

- Chaplain

- Geneticist

- Research nurse and/or assistant

Abbreviations: ALSA, ALS Association; ALS, amyotrophic lateral sclerosis. 


\section{Symptom management: fatigue}

Fatigue is a common first complaint for the person with ALS; researchers estimate that fatigue affects between $44 \%$ and $86 \%$ of all people with ALS. ${ }^{16-18}$ Fatigue is more than just feeling tired; fatigue has been described as feeling an overwhelming exhaustion, having a complete lack of energy. Fatigue in the ALS patient can be both generalized and muscle specific. The cause of fatigue in ALS may be multifactorial, and it can include many factors. Some of these factors and their possible treatments are shown in Table 3.

Fatigue may be related to insomnia that could be multifactorial in origin ("Symptom management: insomnia" section). Lo Coco and La Bella ${ }^{19}$ found in their study of 91 patients with ALS that physical impairment and nighttime complaints (nocturia and muscle cramps) contributed to fatigue, suggesting that treatment of sleep problems may be helpful in lessening fatigue in this population. The nurse should first try to ascertain the probable cause or causes of fatigue in the person with ALS and address each possible factor.

Treatment for fatigue should include energy conservation. Nurses can teach energy conservation to the person with ALS simply by suggesting that he or she use "common sense", with any movement, for example, suggest that the person sit versus stand to complete activities of daily living (ADLs); encourage use of adaptive equipment such as reachers, long-handled shoe horns and sponges, button hooks, shower chairs, and three-in-one commodes; suggest that the patient rest before a desired activity to save their energy for what is important to them (eg, a walk outside).

\section{Symptom management: impaired mobility}

Related to fatigue is impaired mobility. Progression is the word to remember with this illness; it is important for patients, caregivers, and health care professionals to understand that the person with ALS, if he or she lives long enough, will eventually become quadriplegic. Both speed of progression

Table 3 Possible causes and treatments of fatigue in the person with ALS

- Disease progression - energy conservation

- Respiratory insufficiency - raise head of bed, use of noninvasive respiratory devices such as BiPAP or Trilogy

- Side effects from medication, including riluzole - stop or decrease

- Insomnia - "Symptom management: insomnia" section

- Depression - medication, supportive therapy, and complementary and alternative therapies such as acupuncture and massage

Abbreviations: ALS, amyotrophic lateral sclerosis; BiPAP, bilevel positive airway pressure. and site of progression vary from person to person, however. Nurses will need to evaluate the person with ALS for changes in impairment and possible equipment needs over time. In addition, physical and occupational therapists can be consulted, in both the outpatient and home setting, to determine both patient safety concerns and equipment needs.

Table 4 addresses commonly prescribed or suggested equipment for the person with ALS.

Many people when first diagnosed with ALS ask if strengthening exercises can improve muscle weakness. The literature on the benefits of exercise with ALS is inconclusive. ${ }^{20}$ What is agreed upon in the literature is the need for daily stretching and range of motion (ROM) exercises. ROM and stretching exercises can help reduce pain, and can increase or maintain function. ${ }^{21}$ In addition, both ROM and stretching exercises play a role in prevention of contractures, frozen shoulders, deep vein thrombosis, and pulmonary embolism..$^{22,23}$ Nurses, as well as physical and occupational therapists, can teach patients and their loved ones how to do both ROM and stretching exercises.

\section{Symptom management: pain}

Pain is an another common symptom associated with ALS estimates range from $23.9 \%$ to $73 \%$ of ALS patients complain of pain at some point during the course of their disease. ${ }^{15}$ Two common sources of pain depending on the degree of upper or lower motor neuron involvement include:

- Cramps. Often an early symptom, typically described as a "charley horse" type pain. Can occur in all voluntary muscles. There is no FDA-approved, evidence-based drug treatment for cramps, ${ }^{15}$ although anticonvulsant

Table 4 Commonly prescribed/suggested equipment in (often) hierarchical and/or chronological order

For lower extremity weakness:

- Cane (single point or quad)

- Carbon fiber ankle splints (for ankle weakness)

- Walker with 5" front wheels and rear glides

- Companion/travel wheelchair and/or manual wheelchair

- Power wheelchair

For transfers:

- Pivot disc

- Transfer boards

- Mechanical lifts (manual or electric)

Other (possible) equipment:

- Wrist/hand splints

- Shower and tub benches/chairs

- Multipodus boots (to protect heels from skin breakdown)

- Neck collars

- Lift chairs to assist with standing and for comfort 
medication, such as phenytoin, may be of help. Quinine sulfate was used in the past to treat cramps, but has since been removed for the treatment of cramps by the FDA. Tonic water contains quinine; some people with ALS appear to get some relief from cramps by drinking tonic water. In addition, massage and stretching may be of help.

- Spasms. Another early symptom, often described as a tightness, stiffness, or "pulling" type pain. Can occur in all voluntary muscles. Can result in decreased coordination and function. While there is no evidence-based research to support any type of treatment for spasms, ${ }^{15}$ antispasmodics might be of help (including baclofen or tizanidine) along with stretching and massage. In addition, botulinum toxin injections may help relieve spasmtype pain in certain muscle groups.

As the disease progresses, pain is often related to problems of immobility. As ALS does not affect the sensory nerves, the person with ALS is able to feel both pleasure and pain. Lying or sitting in one position will result in the latter.

Nurses need to first assess the source of pain in the person with ALS and intervene/suggest accordingly. For pain related to spasms or cramps, possible interventions have been discussed in the "Symptom management: pain" section. For problems related to general immobility, caregivers should be taught about the need for position changes when the person with ALS is not able to do so on their own. Pillows, mattress pads, and seat cushions can be used on hospital beds and chairs to provide padding. Multipodus boots may help with heel pain while in bed. As previously noted, nurses should teach both the ALS patient and their loved ones how to do ROM exercises, particularly of the shoulder joints to prevent frozen shoulders. Hand splinting may help to prevent finger contractions. Nonnarcotic agents, such as nonsteroidal antiinflammatory medications (eg, Ibuprofen) may help with mild-to-moderate pain. Narcotic analgesics have been shown to be more effective although they can depress respiration, decrease airway protection, and suppress coughing. ${ }^{21}$

\section{Symptom management: respiratory insufficiency}

The respiratory muscles that become weak in ALS are the diaphragm, intercostal muscles, and the accessory muscles of breathing (sternoclei domastoid and scalene muscles). Respiratory muscle weakness in ALS can cause dyspnea and orthopnea as well as sleep disturbance and carbon dioxide retention, which can lead to morning headaches, daytime somnolence, fatigue, and confusion. Since respiratory muscle strength cannot be determined by direct manual muscle testing, a pulmonary function test is most often done in the office setting. ${ }^{21}$ There are limitations to this test, however. Weakness in the bulbar muscles can falsely lower the result as can poor performance due to lack of coordination or understanding. In general, the forced vital capacity result is used as a guide, and the most important "measure" is the symptoms reported by the patient. Another test that can be done is an overnight nocturnal oximetry test. This shows the oxygen level while the person is sleeping. Poor oxygenation for greater than $5 \%-10 \%$ of the night can be an indication of neuromuscular respiratory impairment.

Supplemental oxygen is not the way to treat neuromuscular respiratory insufficiency even if the oxygen saturation is below $90 \%$. People with ALS respiratory insufficiency tend to retain carbon dioxide due to inefficient exhalation and adding supplemental oxygen will potentially increase carbon dioxide to dangerous levels. ${ }^{24}$ Noninvasive ventilation (NIV) is the treatment of choice in ALS respiratory insufficiency.

NIV is delivered via a bilevel positive airway pressure device or via a portable ventilator. Both devices can be used with a mask over the mouth and nose or just at the nose. With the bilevel device, positive pressure or inhalation is prescribed according to the settings called inspiratory positive airway pressure (IPAP). The expiratory positive airway pressure (EPAP) setting is at a much lower amount of pressure to allow for natural exhalation that is facilitated by the degree of inspiratory pressure. In addition, there is a preset rate that delivers the IPAP/EPAP, a determined (by the health care provider) number of sequences per minute. These settings are adjusted as the respiratory muscles weaken and more respiratory support is needed.

A portable ventilator provides both volume and pressure ventilation and delivers the therapy either invasively (via a tracheostomy) or noninvasively (via a mask). The settings are more specific to the needs of respiration and can be more finely tuned than with a bilevel device. A portable ventilator has a 6-hour internal battery and is fairly lightweight. A bilevel device does not have a battery and must be plugged into an electrical outlet or have a car adapter at all times to function. Insurance in the USA is now paying for a portable ventilator when used noninvasively, but it is more costly and can pose a problem if the person is referred to hospice.

Both of these devices, when used noninvasively (with a mask), have limits in their ability to assist with breathing. As the respiratory muscles weaken due to ALS, even these devices cannot sustain life. ${ }^{19}$ Prior to that point, decisions have to be made regarding invasive ventilation via tracheostomy 
versus hospice care. Some people at the end of life may opt to stop using an NIV device to hasten death. This can be done with the guidance of a hospice team and efforts can be made to maintain the person's comfort through the use of medications such as morphine and lorazepam.

The following are some pointers based on the author's experience for care of ALS respiratory symptoms at the end of life.

- Positioning to keep torso straight.

- Maximize use of NIV.

- Move the device from room to room during the day.

- Change masks - often a nasal interface is useful during the day and a fuller face mask for overnight.

- Increase inspiratory pressure as needed-subjectively determined by comfort of patient or based on increasing feeling of shortness of breath.

- Administer anxiolytics and opiates.

- Anxiolytics pills, liquid, concentrate, or topically.

- Concentrated opiates, such as morphine solution - increasing the dose to relieve feeling of air hunger.

- Long-acting opiates with concentrated opiate solution PRN (when necessary).

- Opiate patch.

- Alternative therapies.

- Relaxation and meditation techniques.

- Therapeutic massage.

○ Music.

\section{Symptom management: secretions and airway clearance}

Management of secretions in people with ALS is one of the most difficult symptoms to treat. People with ALS can develop weakness of the bulbar muscles, which is used for speech and swallowing. As a result, handling normal saliva production becomes an impossible task for the muscles of swallowing. ${ }^{25}$

Normally people do not think about swallowing saliva throughout the day. We all produce approximately $1.5 \mathrm{~L}$ of saliva a day. That requires a lot of swallowing. Conversely, the ability to cough and clear thicker secretions such as phlegm can also become a difficult task. This can be further hampered by weak respiratory muscles making coughing ineffective. We also produce a lot of phlegm, some more than others. Phlegm production is influenced by seasonal and environmental allergies and irritants as well as upper respiratory infections and postnasal drip.

Excess saliva is called sialorrhea. There are several pharmacologic choices to treat sialorrhea: ${ }^{25-27}$
- Anticholinergics as tolerated:

○ Hyoscyamine - up to $0.25 \mathrm{mg}$ QID (four times a day)

- Glycopyrrolate - up to $2 \mathrm{mg}$ QID

- Scopolamine patch - one every 72 hours

- Atropine drops $-1 \%$ eye solution - 1-2 drops sublingual every 4-6 hours.

- Botox injections to parotid glands and submandibular salivary glands, every 3 months. ${ }^{28}$

- Tricyclic antidepressants can also be used at low doses for the anticholinergic properties. Examples include amitriptyline, nortriptyline.

- Radiation treatments to parotid glands every 4-6 weeks $\times 3$.

Thick secretions such as phlegm can be treated as follows: ${ }^{29}$

- Adequate fluid intake if possible.

- Quaifenesin 300-600 mg QID.

- Nebulizer with albuterol $0.083 \%$ and acetylcysteine $10 \%$, 2 cc BID (best prescribed in the setting of an adequate cough or with the use of inexsufflator).

- Nasal corticosteroid spray to decrease postnasal drip.

- Suction machine with yankauer catheter to help expectorate secretions.

- Inexsufflator, cough assist machine - mimics a cough with a positive followed by negative pressure for people with neuromuscular respiratory weakness.

\section{Symptom management: dysphagia}

Bulbar weakness leads to dysphagia in people with ALS. It most often begins with difficulty swallowing thin liquids in people with pharyngeal weakness and difficulty chewing and moving food in the mouth in people with oral weakness. ${ }^{21}$ Assessment by a speech pathologist (SLP) and consultation with a dietitian (RD) are both necessary for the person with dysphagia. The SLP will determine what food and liquid consistencies are safest to consume along with teaching safe swallowing strategies. The RD will recommend food choices and how to maintain caloric intake to prevent weight loss. The SLP and RD in conjunction with the physician will determine when a gastrostomy tube is needed due to increased risk of aspiration. ${ }^{30}$

There are suggestions that the SLP and RD will make to help the person with dysphagia.

- Thicken thin liquids with Thick-It (Precision Foods, St Louis, MO, USA).

- Drink natural nectar thick liquids.

- Avoid mixed consistencies such as vegetable soup that has solids and liquid in one mouthful.

- Smaller, more frequent meals. 
- Softer foods such as mashed potatoes, yogurt, fish, chopped chicken, oatmeal.

- Do not shy away from higher calorie foods such as whole milk, gravy, cheese.

- Add nutritional supplement drinks.

- Avoid dry, crumby foods, and food with small pieces such as rice.

Strategies for safe swallowing should also be taught to the person with dysphagia as well as the caregiver.

- Use of chin tuck when swallowing (if recommended by the SLP, not always useful for everyone with dysphagia).

- Avoid distractions when eating, such as conversation or watching TV. Concentrate on safe swallowing strategies.

- Administer medications one at a time in a spoonful of applesauce or yogurt. Some pills can be crushed and given this way also.

- Caregivers should be taught the Heimlich maneuver.

- Monitor weight, if possible and report loss to your health care practitioner.

Loss of weight and progression of ALS are linked even when weight loss is not associated with dysphagia. ${ }^{31}$ It is important to provide this information in discussions with patients regarding calorie supplementation and decisions regarding a gastrostomy tube.

The need for a gastrostomy tube is determined by degree of weight loss, length of time it takes to eat a meal, and signs of aspiration such as increased choking, coughing, and pneumonia. The discussion of whether to get a gastrostomy tube is one that the person with ALS, the family, and the health care practitioner will need to have. The procedure is often performed in same-day surgery via an endoscope. This type of feeding tube is called a peg tube (percutaneous endoscopic gastrostomy tube). Conscious sedation is used to perform the (peg tube) procedure, so the degree of respiratory compromise has to be taken into consideration. If the person with ALS has respiratory insufficiency, then a gastrostomy tube can be inserted in the Interventional Radiology department with local anesthesia while on NIV.

Some people at the end of life may opt to stop tube feedings to hasten death. This can be done with the guidance of a hospice team, and efforts can be made to maintain the person's comfort. Caloric needs and tolerance of food (by either mouth or feeding tube) can greatly diminish at the end of life and should not be forced beyond the person's comfort. Complications include early satiety, reflux, poor gastric emptying, constipation, or diarrhea. All of these symptoms can be managed by hospice in conjunction with the health care practitioner.

\section{Symptom management: dysarthria}

Bulbar weakness leads to dysarthria in people with ALS. In addition to difficulty speaking, low vocal volume can be a problem with respiratory insufficiency and make it difficult to hear what the person is saying. Communication is such a vital part of life for safety as well as QoL reasons. ${ }^{21}$ It is important to provide a means of communication.

For people with dysarthria, there are premade letter boards that can be obtained from an ALSA, or the family can make a letter/picture board. The letter or picture is pointed to so that a word can be spelled or phrase indicated. Some communication boards are divided into quarters with each section of the alphabet in each quarter. They are designed for the caregiver to hold and the person with ALS to look at which quarter holds the letter or phrase they want to say and the caregiver recites each letter or phrase in that quarter to sort out the message. It is a tedious process, but people get very quick using it after some practice. Another means to communicate is typing on a keyboard on a computer or tablet or by writing. This is very useful for people with use of a hand/arm. Some people attach a laser pointer to a head band and use it to point to a letter/picture board on the wall. Furthermore, there are eye gaze systems for people who have no use of their upper extremities. These are often available through insurance as a rental item.

Most of the high-tech options are now available via tablet and computer apps and programs. People can be directed to record their own voice (voice banking) early on in the disease process so their voice can be used with devices or apps that speak for them.

For people with low vocal volume due to respiratory insufficiency, there are some strategies that can help with effective communication. The caregivers need to be aware of the person's inability to project his/her voice, so being present, ideally in front of the person, when he/she speaks is best. Having a call device nearby to signal the need to communicate face-to-face is helpful. Use of a voice amplifier can also help; these are available online. Using some of the techniques and strategies for people with dysarthria can also conserve energy for people with respiratory compromise.

\section{Symptom management: laryngospasm}

Laryngospasms can be very frightening, but are almost never life-threatening. A laryngospasm occurs when something 
triggers the vocal cords to close suddenly making it difficult to breathe or speak. ${ }^{30}$ It usually corrects itself within seconds, but causes coughing and anxiety that make the spasm worse. Laryngospasms in the ALS patient can occur secondary to upper motor neuron involvement of the bulbar muscles, and/or reflux of stomach acid into the esophagus. The first line of treatment for laryngospasms is to instruct the person to try and remain calm, breathe through the nose, and know it will pass momentarily. It is important to treat reflux with diet modification and medication. ${ }^{32}$ In the cases of upper motor neuron involvement, use of clonazepam can be helpful. Clonazepam is sedating, so it is best to start with a low night time dose.

Another upper motor neuron manifestation can be jaw clenching and possible dislocation of the temporomandibular joint. ${ }^{33,34}$ This can also be a painful and frightening experience. Jaw clenching can cause inadvertent biting of the tongue or inside of the cheeks. If this biting occurs, treating the sore areas with glyoxide is helpful to soothe and heal the area. Using a mouth guard when sleeping also helps prevent jaw clenching. Baclofen or clonazepam may be helpful with jaw clenching also. If use of a mouth guard and medication have not helped, then botox injections can be considered.

\section{Symptom management: depression/anxiety}

The prevalence of clinical depression and ALS has been noted to be between $2 \%$ and $75 \%{ }^{21}$ It is unclear why there is such a wide margin among the various research studies that look at these combined illnesses. What is to be expected with the diagnosis of ALS - most patients will experience a reactive depression or grief. Education and supportive therapy can be of help with those who are dealing with grief. Clinical depression is associated with poor QoL in people with ALS and should be treated aggressively with both antidepressants and supportive therapy.

Anxiety can be difficult to recognize in the person with ALS; both anxiety and dyspnea can result in similar complaints (eg, shortness of breath, feeling of impending doom, rapid heart rate). Montel et $\mathrm{al}^{35}$ found that anxiety and somatic disorders increased over time in their ALS population; the authors suspected both physical deterioration and fear of death as factors that exacerbated or caused anxiety in the patients they studied. Ozanne et $\mathrm{al}^{36}$ noted that anxiety in their (ALS) population was related to fear of dying in distress (choking to death or gasping for breath). As with depression, anxiety is often a treatable illness. Antidepressants with or without anxiolytic medications may be of help. It is important for nurses to educate patients and their loved ones about the dying process and symptom management. Supportive therapy that involves dealing with existential questions and concerns should be offered. Spiritual despair should be addressed by appropriate spiritual and religious counselors.

\section{Symptom management: insomnia}

Insomnia in the person with ALS may be caused by one or more of the following:

- Pain - spasms, cramps

- Depression and/or anxiety

- Immobility - being unable to turn in bed

- Respiratory insufficiency.

Treatment of insomnia in this patient population is based on (probable) cause or causes; nurses should assess for both probable causes along with contributing factors. As noted above, pain should be treated by appropriate medication, along with stretching and ROM exercises. Depression and anxiety need to be acknowledged and treated. The person with ALS who is immobile while in bed needs to be turned! This can cause caregiver issues/problems as the caregiver needs to sleep too. Raising the head of the bed may help with early respiratory insufficiency. In addition, some people with ALS find it more comfortable to sleep in their power wheelchairs or lift chairs; both chairs provide (some) trunk support along with the ability to raise the head and trunk of the chairs.

\section{Symptom management: pseudobulbar affect}

Pseudobulbar affect, or uncontrollable laughing or crying, can be a troublesome symptom for the person with ALS. Although not a mood disorder, this symptom has been treated with antidepressants, in particular selective serotonin reductase inhibitors, with some effectiveness. ${ }^{21}$ A newer drug, Nuedexta ${ }^{\circledR}$ (Avanir Pharmaceuticals, Aliso Viejo, CA, USA), has been shown to be effective in treating pseudobulbar affect in this patient population, according to the 2009 Neurology Practice Parameter update group,,${ }^{15}$ and is generally well-tolerated.

\section{Symptom management: cognitive issues}

As previously noted, the CI that is related to ALS ranges from mild (CI) to severe (FTD). While nursing interventions need to reflect where the ALS patient is on the CI scale, some general caregiver interventions are as follows:

- Educate the caregiver about CI/FTD, in particular, explain the organic nature of the illness (that it is an illness, person cannot help his or her $\mathrm{CI} /$ behavior). 
- Address safety and environmental concerns, including medication management, driving, managing finances, use of power tools/equipment, keeping anything harmful out of sight/reach.

- Address (possible) behavioral concerns by using a calm voice, giving one-step directions, avoid trying to reason with the patient, supervising activities such as eating and bathing, finding and utilizing soothing activities.

- Advise of the need for the person with ALS to complete his or her Advanced Directive and Living Will. The patient should also identify and ensure a legal medical power of attorney; this person would speak on the behalf of the person with ALS (re: future medical care, if the person with ALS is unable to do so).

\section{Caregiver issues}

It is not surprising that a study involving 800 caregivers of patients with ALS demonstrated declining physical and mental health well-being scores over time. ${ }^{37}$ Another study demonstrated that QoL for caregivers was lower than for patients with ALS. ${ }^{38}$ Caregivers of people with ALS are faced with many challenges, particularly related to the progressive nature of the disease. A person with ALS will get weaker and weaker requiring more and more help; eventually the person with ALS will need total care with all ADLs. Equipment will most likely be utilized, including walkers, wheelchairs, hospital beds and patient lifts, Bilevel positive airway pressures or Trilogy's machine, nebulizers, and inexsufflators. The person with ALS may require feedings via a gastrostomy tube. He or she may utilize equipment to communicate, such as a letterboard or computer. The caregiver, who is most likely "not" going to be a registered nurse or other health care professional, needs to be instructed in all aspects of care.

In addition to the physical care of the person with ALS, the caregiver may also have to face other challenging issues. There may be financial concerns due to loss of income, cost of equipment, and cost of in-home care. There may be children at home and/or other family members in need of the caregiver's attention, such as an elderly parent. The person with ALS may also have CI or even FTD.

\section{Nursing considerations}

The registered nurse caring for the person with ALS and their loved ones needs to be aware of the following: the progressive nature of the illness, the (at times) problematic symptoms of the disease, current treatment of symptoms, and the possible cognitive component associated with ALS. The nursing care plan should reflect interventions that are family-focused versus specific to the patient; support and education for the caregiver should be included in the plan. In addition, the nurse should investigate potential community supports that may be of help to the family, eg, local ALS Chapter, home care, hospice, and palliative care programs.

\section{Summary}

The reality of ALS is that it is both progressive in nature and eventually, in most cases, fatal. Palliative care, which involves symptom management, is the usual treatment of choice for those diagnosed with ALS. Nurses can be instrumental in providing education and care to both the ALS patient and their caregivers.

\section{Disclosure}

The authors report no conflicts of interest in this work.

\section{References}

1. Miller RG, Jackson CE, Kasarskis EJ, et al. Practice parameter update: the care of the patient with amyotrophic lateral sclerosis: drug, nutritional, and respiratory therapies (an evidence-based review). Neurology. 2009;73(15):1218-1226.

2. Mitsumoto H. The clinical features and prognosis ofALS. In: Mitsumoto H, Munsat TL, editors. Amoytrophic Lateral Sclerosis. New York, NY: Demos; 2001:345.

3. ALSA.org [Internet]. Facts you should know. Washington, DC: The ALS Association 2015. Available from: http://www.alsa.org/about-als/ facts-you-should-know.html. Accessed October 10, 2015.

4. Mehta P, Antao V, Kaye W, et al. Prevalence of amyotrophic lateral sclerosis - United States, 2010-2011. MMWR Morb Mortal Wkly Rep. 2014;63(7):1-14.

5. ALS.org [Internet]. ALS in the military: unexpectant consequences of military service. February 2013. Available from: http://www.alsphiladelphia. org/document.doc?id=2011. Accessed October 23, 2015.

6. Lomen-Hoerth C, Murphy J, Kramer RK, Olney RK, Miller B. Are amyotrophic lateral sclerosis patients cognitively impaired? Neurology. 2003;60:1094-1097.

7. Ringholz GM, Appel SH, Bradshaw M, Cooke NA, Mosnick DM, Schulz PE. Prevalence and patterns of cognitive impairment in sporadic ALS. Neurology. 2005;65(4):586-590.

8. Machts J, Bittner V, Kasper E, et al. Memory deficits in amyotrophic lateral sclerosis are not exclusively caused by executive dysfunction: a comparative neuropsychological study of amnestic mild cognitive impairment. BMC Neurosci. 2014;15:83.

9. Hu WT, Shelnutt M, Wilson A, et al. Behavior matters - cognitive predictors of survival in amyotrophic lateral sclerosis. PLoS One. 2013;8(2):e57584.

10. Goldstein L. Control of symptoms: cognitive dysfunction. In: Oliver D, Borasio GD, Walsh D, editors. Palliative Care in Amyotrophic Lateral Sclerosis from Diagnosis to Bereavement. New York, NY: Oxford; 2006:111-127.

11. Neary D, Snowden JS, Mann DM. Cognitive change in motor neuron disease/amyotrophic lateral sclerosis (MND/ALS). J Neurol Sci. 2000;180:15-20.

12. Lepow L, Van Sweringen J, Strutt AM, et al. Frontal and temporal lobe involvement on verbal fluency measures in amyotrophic lateral sclerosis. J Clin Exp Neuropsychol. 2010;32(9):913-922. 
13. Moss AH, Casey P, Stocking CB, Roos RP, Brooks BR, Siegler M. Home ventilation for amyotrophic lateral sclerosis patients: outcomes, costs, and patient, family and physician attitudes. Neurology. 1993; 43(2):438-443.

14. Bromberg MD. Life support: realities and dilemmas. In: Mitsumoto H, Monsat TL, editors. Amyotrophic Lateral Sclerosis. New York, NY: Demos; 2001:196.

15. Miller RG, Jackson CE, Kasarskis EJ, et al. Practice parameter update: the care of the patient with amyotrophic lateral sclerosis: multidisciplinary care, symptom management, and cognitive/behavioral impairment (an evidence-based review). Neurology. 2009;73(15):1227-1233.

16. Lou JS, Reeves A, Benice T, Sexton G. Fatigue and depression are associated with poor quality of life in ALS. Neurology. 2003;60: $122-123$.

17. Ramirez C, Piemonte ME, Callegaro D, Da Silva HC. Fatigue in amyotrophic lateral sclerosis: frequency and associated factors. Amyotroph Lateral Scler. 2008;9:75-80.

18. McElhiney MC, Rabkin JG, Gordon PH, Goetz R, Mitsumoto H Prevalence of fatigue and depression in ALS patients and change over time. J Neurol Neurosurg Psychiatry. 2009;80:1146-1149.

19. Lo Cocco D, La Bella V. Fatigue, sleep, and nocturnal complaints in patients with amyotrophic lateral sclerosis. Eur J Neurol. 2012;19: $760-763$.

20. Lewis M, Rushanan S. The role of physical therapy and occupational therapy in the treatment of amyotrophic lateral sclerosis. NeuroRehabilitation. 2007;22:451-461.

21. Simmons Z. Management strategies for patients with amyotrophic lateral sclerosis from diagnosis through death. Neurologist. 2005;11(5): 257-270

22. Jackson CE, Bryan WW. Amyotrophic lateral sclerosis. Semin Neurol. 1998;18:27-39.

23. Kimura F, Ishida S, Furutama D, et al. Wheelchair economy class syndrome in amyotrophic lateral sclerosis. Neuromuscul Disord. 2006;16:204-207.

24. Bourke S. Respiratory involvement in neuromuscular disease. Clin Med. 2014;14(1):72-75.

25. Hockstein NG, Samadi DS, Gendron K, Handler SD. Sialorrhea: a management challenge. Am Fam Physician. 2004;69:2628-2634.

26. Bei TJ. Management of drooling. Semin Pediatr Neurol. 2003;10: 265-270.
27. MPR Nurse Practitioners' Edition. New York, NY: Haymarket Media, Inc. Summer 2015.

28. Lipp A, Trottenberg T, Schink T, Kupsch A, Arnold G. A randomized trial of botulinum toxin A for treating drooling. Neurology. 2003;61: 1279-1281.

29. Chatwin M, Ross E, Hart N, Nickol AH, Polkey MI, Simonds AK. Cough augmentation with mechanical insufflation/exsufflation in patients with neuromuscular weakness. Eur Respir J. 2003;21:502-508.

30. Desport JC, Preux PM, Truong CT, Courat L, Vallat JM, Couratier P. Nutritional assessment and survival in ALS patients. Amyotroph Lateral Scler Other Motor Neuron Disord. 2000;1(2):91-96.

31. Körner S, Hendricks M, Kollewe K, et al. Weight loss, dysphagia and supplement intake in patients with amyotrophic lateral sclerosis (ALS): impact on quality of life and therapeutic options. BMC Neurol. 2013;13:84

32. Obholzer RJ, Nouraei SAR, Ahmed J, Kadhim MR, Sandhu GS. An approach to the management of paroxysmal laryngospasm. J Laryngol Otol. 2008;122:57-60.

33. Hu L, Liao Z, Wang Y, Liao L, Lai W. Efficacy of botulinum toxins on bruxism: an evidence-based review. Int Dental J. 2012;62:1-5.

34. Saletu A, Parapatics S, Anderer B, et al. On the pharmacology of sleep bruxism: placebo-controlled polysomnographic and psychometric studies with clonazepam. Neuropsychobiology. 2005;51:214-225.

35. Montel S, Albertini L, Desnuelle C, Spitz E. Evolution of quality of life, mental health, and coping strategies in amyotrophic lateral sclerosis: a pilot study. J Palliat Med. 2012;15(11):1181-1184.

36. Ozanne AO, Graneheim UH, Strang S. Finding meaning despite anxiety over life and death in amyotrophic lateral sclerosis patients. J Clin Nurs. 2013;22:2141-2149.

37. Wooley J, Ringel S. Caregiver quality of life in ALS: relationship between patient health status and caregiver health-related quality of life, role satisfaction, and workforce participation [abstract]. In: Proceedings of the Eighth International Symposium on Amyotrophic Lateral Sclerosis and Motor Neuron Disease, Glasgow, Scotland, November 1997. Amsterdam, Netherlands: Elsevier; 1998:33.

38. Jenkinson C, Fitzpatrick R, Brennan C, et al. Evidence for the validity and reliability of the ALS assessment questionnaire: the ALSAQ-40. Amyotroph Lateral Scler Other Motor Neuron Disord. 1999;1: $33-40$.
Nursing: Research and Reviews

\section{Publish your work in this journal}

Nursing: Research and Reviews is an international, peer-reviewed, open access journal publishing original research, reports, reviews and commentaries on all aspects of nursing and patient care. These include patient education and counselling, ethics, management and organizational issues, diagnostics and prescribing, economics and

\section{Dovepress}

resource management, health outcomes, and improving patient safety in all settings. The manuscript management system is completely online and includes a very quick and fair peer-review system. Visit http://www.dovepress.com/testimonials.php to read real quotes from published authors. 